\title{
Achieving Fairness in IEEE 802.11 Ad Hoc Networks
}

\author{
Fanilo Harivelo and Pascal Anelli \\ IREMIA, Université de La Réunion \\ BP 7151, 15 Avenue R. Cassin, 97715 Saint Denis Messag 9, France \\ \{Fanilo.Harivelo | Pascal.Anelli\}@ univ-reunion.fr
}

\begin{abstract}
IEEE 802.11 has become the main technology in local area wireless networks. However, performance anomalies, especially, in terms offairness, arise in its use in ad hoc networks. This paper introduces a fair access method (FWM: Fair Wireless MAC) that is an extension of the original 802.11 DCF access method to ad hoc networks. A busy tone-like mechanism is coupled with carrier sensing in order to synchronize the state of neighboring nodes of a communication. By extending the knowledge of the neighborhood, the distributed contention resolution mechanism of 802.11 can apply again. This paper also deals with unfairness caused by wireless transmission. The evaluation of the FWM protocol is demonstrated through NS-2 simulations in unfair scenarios.
\end{abstract}

\section{Introduction}

A Mobile Ad-hoc Network (MANET) is a selfconfiguring network of mobile nodes connected by wireless links. The resulting network forms an arbitrary topology. Nodes are free to move randomly and organize arbitrarily; thus, the network's wireless topology may change rapidly and unpredictably. Different communication modes can be met for these networks. The simplest one implies direct link communication in which source and destination interact directly. A more complex mode involves nodes allowing multi-hop communications: intermediate nodes relay the transmission from the source to the destination. Independently of the chosen communication mode, transmission lays down on IEEE 802.11 standard [11], commercially called Wireless Fidelity (Wi-Fi). It defines physical and Medium Access Control (MAC) layers. The most widely used access method in a MANET is based on Wi$\mathrm{Fi}$ access method working according to a contention-based mode known as Distributed Coordination Function (DCF). In common Wi-Fi networks, the topology is centered on a node, known as Access Point (AP), and network commu- nications pass through that node. In Fig. 1(a), when two nodes $S_{1}$ and $S_{2}$ communicate with the AP (namely $D$ ) while being out of range of each other, DCF provides a way for the AP to coordinate transmission and avoid collision. This situation is known as the hidden station problem. However, DCF usage in ad hoc mode leads to new issues. As shown in Fig. 1(b), communications are not centered anymore on a node: two receivers $D_{1}$ and $D_{2}$ are involved. Emitters coordination cannot longer be possible as no receiver is within the transmission range of both emitters. This kind of situation leads to unfairness in medium access. Indeed, it has been reported in [5] that under certain situations, fairness issues appear in the use of DCF in ad hoc networks.

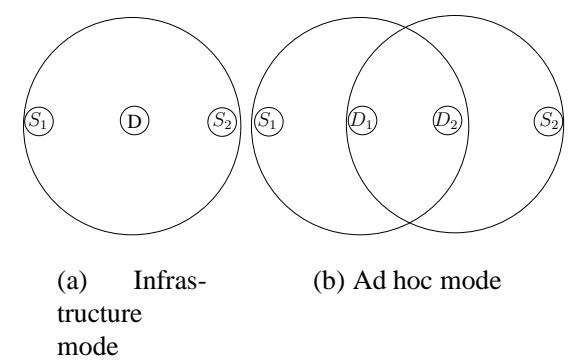

Figure 1. Operating modes of wireless networks

Fairness is of important concern in wireless networks in which resources are limited and channel condition highly dynamic. In unfair configuration, not only is the traffic condition involved in resources sharing, but also the relative location of nodes affects the effective utilization of these resources. Therefore, it is difficult to manage resources in such situations. We show that the main causes of unfairness phenomena come from an incomplete knowledge of neighborhood activities (or a lack of synchronization) for nodes. In wireless networks, for a successful transmission through 
direct link, coordination of both the emitter and the receiver is necessary. DCF provides a mechanism to fulfill such a requirement, but due to the difference between sensing range and transmission range [14], it becomes inefficient. Fairness issues are due to the inappropriate execution of DCF in ad hoc mode. The proposed solution is based on DCF; we adapt it to operate in situations exhibiting fairness issues. The independence on the topology, self-configuration, and implementation simplicity motivate the choice of our approach. The principle consists in introducing a signaling channel, exploiting the busy tone scheme [16], in addition to the working channel running DCF. The proposition, called Fair Wireless MAC (FWM), uses the signaling channel to inform neighbors of an ongoing communication. In addition to carrier sensing, a node performs access method by also considering the signaling channel. Evaluations of this solution show that unfairness still remains due to varying waiting delays for nodes trying to access the medium. Then, FWM comprises an additional mechanism for the synchronization of the beginning of the waiting period .

The paper will be organized as the following. Section 2 presents the DCF IEEE 802.11 mechanism and its fairness limitations in ad hoc networks. The design of the proposed solution is detailed in section 3 . We study two basic scenarios; the integration of the solution with DCF and its efficiency are tested with these scenarios. Section 4 presents the performance evaluation of the solution with other scenarios that are the combination of the basic cases, including the chain topology. Section 5 presents existing solutions as well as their drawbacks and limitations. Section 6 ends the paper.

\section{Fairness issues in DCF}

DCF function is the main access method of the IEEE 802.11 standard [11]. It is based on the CSMA/CA mechanism. Carrier-sensing is done both at the physical layer level and at the MAC layer level. At the physical layer, sensing is carried out by detecting the activity of other nodes on the channel. At the MAC level, this is done with the help of the duration of the current transmission, announced in the frame header. When receiving this information, stations update their Network Allocation Vector (NAV). That duration includes a Short Inter-Frame Space (SIFS) and the acknowledgment transmission delay. A station having a frame to transmit can initiate its transmission if the medium is idle for more than a DCF IFS (DIFS). If the medium is busy, the station delays its transmission until it becomes idle again during a DIFS and computes a backoff random value. The backoff timer is decreased as the channel is idle, frozen if it is busy, and resumed once the channel becomes free again for a duration greater than DIFS. A station can initiate its transmission if its timer reaches zero. The backoff value is chosen randomly in the interval $[0, w-1]$, called CW (Contention Window). In case of transmission failure, the value of $w$ is doubled up to a maximum value $2^{m} W=C W_{\max }+1$ where $W=C W_{\min }+1$. On receipt of a frame, the destination node waits for a SIFS and sends an acknowledgment (ACK) to the source to indicate the frame was correctly received. If the frame is lost or if the transmitting station does not receive an acknowledgment, the value of the $w$ is doubled. When an emitter receives an erroneous frame that cannot be decoded, it defers its transmission of an Extended IFS (EIFS) (longer than a DIFS) interval time instead of a DIFS, when the medium becomes idle. This is to protect ACK transmission. EIFS value is slightly greater than 7 DIFS. DCF has the benefit to be flexible and easy to deploy. It then becomes the de facto access method for MANETs.

Signal attenuation depending on the distance constitutes one of the specificities of 802.11. A received signal may be impossible to decode beyond a certain distance. The transmission range $r_{t}$ defines the area within which a packet can be successfully received while the sensing range $r_{s}>r_{t}$ defines the area within which a transmission can be sensed but the content may not be received correctly.

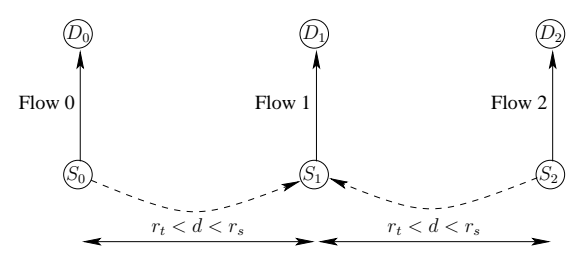

(a) Unbalanced contention

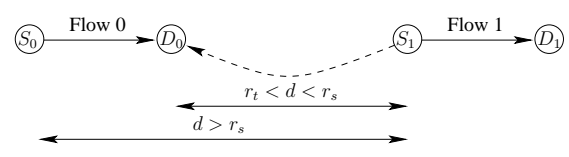

(b) Hidden station

\section{Figure 2. Unfair scenarios}

In addition to the range differences, other problems of medium access fairness also exist. Fig. 2 depicts considered unfair scenarios; pairs are at a distance $d>r_{t}$. In an ad hoc network, all unfairness phenomena are due to the following cases or to a combination of them: (1) the unbalanced level of contention (or double contention area problem), illustrated by the three-pairs scenario in Fig. 2(a), in which the middle flow undergoes more contention than the other flows. In Fig. 2(a), node $S_{1}$ (flow 1) waits for both nodes $S_{0}$ and $S_{2}$ (flows 0 and 2) to stop transmitting to get a successful transmission. While nodes $S_{0}$ and $S_{2}$ are not 
aware of the existence of each other, they will overflow the channel, thus starving node $S_{1}$. (2) the well-known hidden station problem, depicted in Fig. 2(b), in which the traffic for the receiver $D_{0}$ (flow 0 ) is corrupted by the transmission of node $S_{1}$ (flow 1) [7]. In a 802.11 world, a RTS/CTS handshake was designed to overcome this problem within a network configuration like Fig. 1(a). In this scheme, the transmitter sends a RTS packet to the receiver. All neighboring nodes overhearing this packet must defer their transmission according to the expected duration of the communication. When RTS arrives at the receiver, it replies with a CTS packet in which it inserts the duration of the communication so that the node's neighbors defer their transmission as well. It is only on receipt of a CTS that the sender may transmit the data packet. However, such a scheme becomes inefficient in a configuration like in Fig. 2(b), because the interferer transmitter (i.e. node $S_{1}$ ) is out of the transmission range of the receiver (i.e. node $D_{0}$ ) that may therefore not decode the received CTS.

The fairness level is measured by the throughput of sources in the network. All sources are identical and of CBR (Constant Bit Rate) type with constant packet size. The sending rate of a single source is sufficiently high to overload the link. We assume that the capture effect does not exist (i.e. when two or more signals overlap at the receiver, it considers that a collision occurs without considering each signal power). The design of the proposed solution is presented in the following and verified by simulation in NS-2 using the topologies depicted in Fig. 2.

Table 1 shows the simulation results for the basic scenarios with a classical ad hoc network exhibiting unfairness between nodes and flows. In scenario 2(a), node $S_{1}$ (flow 1) has to wait for both nodes $S_{0}$ and $S_{2}$ (flows 0 and 2) to stop sending before accessing the channel. Nodes $S_{0}$ and $S_{2}$ are not in the sensing range of each other. In scenario 2(b), node $S_{1}$ is not aware of the existence of node $S_{0}$ and generates greedily, harming the receipt of flow 0 at node $D_{0}$. Thus, flow 0 can not even experience a successful transmission, and does not appear in the graphic.

These two problems can be viewed as an incomplete knowledge of the neighborhood activities or a lack of synchronisation. The following section aims at extending DCF to handle the ad hoc operating mode.

\section{Design of a fair access method}

The problems identified in the previous section are addressed in the following, as well as their integration into 802.11 DCF.

\subsection{Incomplete knowledge of the neighbor- hood activities problem}

Hidden station and unbalanced contention problems do not appear if the state of each node is propagated to its neighborhood. The state of a node is defined by the ongoing communication. Thus, the emitter is in a sending state. The receiver is in a receiving state, either it is the destination of the communication or not. Otherwise, no communication is present and the node is in the idle state. As the access method is carrier sense-based, the neighbors of emitter are aware of its sending state, thus this state is taken into consideration in the access method procedure itself. For instance, in the three-pairs scenario (Fig. 2(a)), the two side couples know when the center couple is transmitting. The problem resides in the receiving state of the node. Indeed, in a direct link ad hoc configuration, some neighbors of a node in receiving state are not informed of this situation. For instance, in Fig. 2(a), node $S_{0}$ is not aware that node $S_{1}$ is receiving from node $S_{2}$ (because node $S_{1}$ cannot transmit while the channel is occupied by node $S_{2}$ ), and so is node $S_{2}$ for the transmission of node $S_{0}$. The same observation is made in Fig. 2(b) for transmission from node $S_{0}$. Thus, the missing propagation of node state involves the receiving state. Then, an incomplete knowledge of neighborhood activities may explain fairness issues.

The propagation of the receiving state of each node to its neighbors constitutes a natural way to fix the hidden station and the unbalanced contention issues. In infrastructure mode as depicted in Fig. 1(a), the RTS/CTS handshake in 802.11 aims at broadcasting to neighbors of the sender and the receiver the information indicating that a communication will take place between them, and reserves the medium along the transmission period. However, in ad hoc mode, this mechanism is no longer efficient as some nodes cannot interpret or receive control packets indicating a receiving state. In Fig. 2(b), node $S_{1}$ cannot decode the CTS sent by node $D_{0}$ as it is out of its transmission range. RTS/CTS mechanism aims to ensure synchronization at MAC level as far as nodes are within transmission range of each other. Once they are located out of the transmission range, this approach is no longer relevant. Thus, the receiving state of a node should be deduced by a simpler signal which is present along the duration of the communication. As this signal cannot be transmitted in the same channel as that used by the emitter, then it should require another channel. In this case, synchronization is done at the physical level. The additional channel purpose is for out-of-band signaling only. The signal on this signaling channel is of busy tone type (existence or absence of a signal). Initially, the busy tone scheme was proposed in [16] as an access method that tackles with hidden station and exposed station problems. In the DCF context, the busy tone signal will serve for a node 


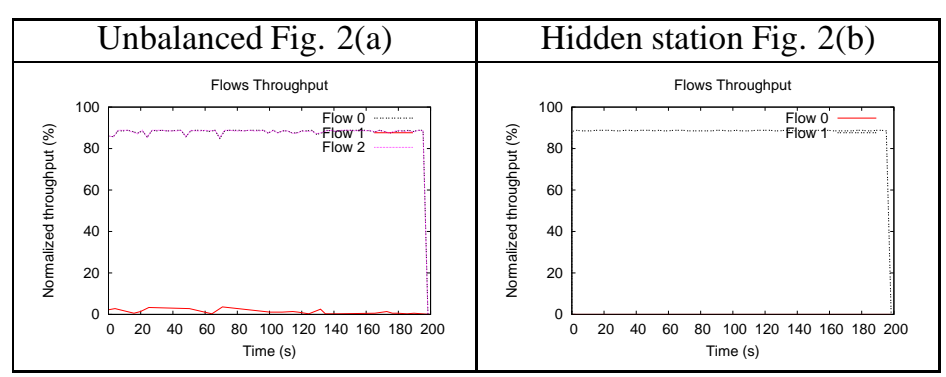

\section{Table 1. Flows throughput with classical DCF}

to indicate that it is in receiving state. Thus, a node keeps on generating a signal as long as it receives data from other nodes and stops the signaling when the channel becomes idle. The node generates a busy tone signal even if it is not the destination of the packet in order to prevent an unadvertised node from starting a new communication.

The integration of these functionalities in legacy IEEE 802.11 DCF concerns: (1) The introduction of a signaling channel. It is worth noticing that the signaling channel is only informative and, a narrow band of frequency is sufficient. (2) An additional condition for the carrier sensing: any node in the network considers both the working channel state and the signaling channel state during carrier sensing phase. That is, a node considers the medium to be idle only if the two channels are idle. Then, the backoff timer will resume depending on the inactivity of both channels.

In this case, both side transmitters of Fig. 2(a) are aware of the existence of each other. Indeed, the central pair propagates its receiving state and then, the activity of a side pair to the other side pair. It can be viewed as establishing a single medium shared by the three pairs. Similarly, the hidden aggressive transmitter of Fig. 2(b) senses the communication of the neighboring flow thanks to the signaling channel. Indeed, the DCF access method considers the medium busy during sensing period when a busy signal is detected on the channel. Table 2 shows the results of the evaluation of the proposed changes in DCF for the two considered scenarios.

In scenario (a), unfairness is less severe but still remains. This can be explained by the fact that the two side transmitters are aware of the existence of each other when node $S_{1}$ generates a busy signal while receiving transmission from one of the two other transmitters. However, each successful transmission results in an ACK not being decoded by the middle pair, namely node $S_{1}$, that generates an EIFS. Then, the medium access of node $S_{1}$ is biased because when one side pair finished its transmission, node $S_{1}$ waits for an EIFS and the other side pair accesses the channel. In scenario (b), node $S_{1}$ is aware of the existence of flow 0 thanks to the generation of a busy signal when node $D_{0}$ receives a transmission from node $S_{0}$. However, contrary to the previ- ous subsection, it turns out that node $S_{1}$ (flow 1) now suffers from unfairness from flow 0 . Indeed, when node $D_{0}$ finishes to receive a packet, it sends an ACK to node $S_{0}$ that is also received by node $S_{1}$. The latter cannot decode the received ACK and thus, triggers an EIFS. Therefore, node $S_{0}$ accesses the medium more often.

\subsection{Difference of ranges or EIFS problem}

In ad hoc configuration, the EIFS issue leads to different waiting period start times of the transmitters. A solution consists in equalizing waiting periods. That can be accomplished using one of the following strategies: (1) Prevent the triggering of EIFS, (2) Activate EIFS for all nodes in contention when a node triggers EIFS.

The utility of EIFS delay can be reconsidered in the current context. In 802.11, it is used to keep an ongoing transmission safe from interferences of a node that is not aware of the duration of the communication. This node is then desynchronized with the current transmission. The 802.11 committee chose to have a single channel in the access method. Then, the resynchronization process must be done on temporal basis, namely, with the EIFS waiting delay. However, unfairness phenomena that it conveys are reported in the previous subsection and in [5]. With the existing signaling channel serving to synchronize the receiving state of nodes, a resynchronization with EIFS seems redundant.

Following this line of thought, Table 3 shows the evaluation of the hidden station scenario with the signaling channel and the suppression of EIFS delay. The results exhibit an improvement in fairness level, however, unfairness phenomena still remain. This can be explained by the fact that the two transmitters do not detect the end of flow 1 at the same instant. Indeed, node $S_{0}$ considers the medium to be idle when node $D_{0}$ stops sending in the signaling channel. It does not hear the ACK transmitted by $D_{1}$. Node $S_{0}$ launches its medium access period while node $S_{1}$ is still receiving the ACK. This difference in the beginning of medium access process gives node $S_{0}$ advantage over node 


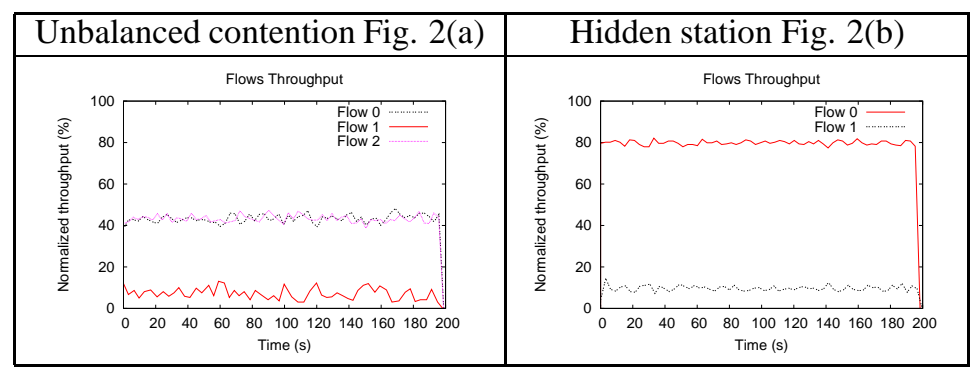

Table 2. Flows throughput with signaling channel

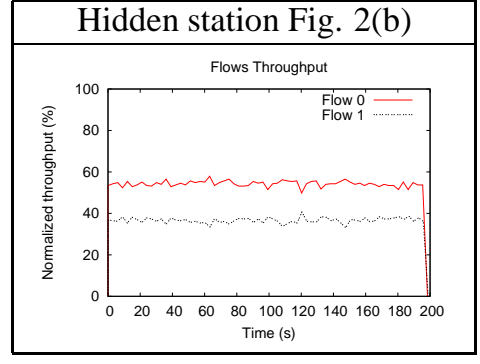

Table 3. Flows throughput with signaling channel without EIFS (Fig. 2(b))

$S_{1}$. It consists in the duration of a SIFS and the transmission of an ACK and equals approximately $314 \mu \mathrm{s}$ (an EIFS equals $364 \mu \mathrm{s}$ ). However, no problem appears for the transmission of flow 0. As shown in Table 3, this leads to an unfair situation in favor of flow 0 . Then, the solution of suppressing EIFS is not efficient, since it must be kept in order to balance the waiting procedure.

The principle of the second alternative consists in equalizing the waiting delay. When a EIFS is triggered at a node, the competitor nodes have to act similarly. That eliminates the channel access bias introduced by frequent transmission deferments due to undecodable packets. A synchronization signal is emitted when an EIFS delay is activated within a node. The implementation of this strategy in physical layer can be either an additional dedicated channel or use of the signaling channel with the signal type deduced from its duration. One notes that a short signal or an impulse is sufficient for the EIFS synchronization. All emitters in the neighborhood hearing this signal also defer their transmission by engaging an EIFS waiting period. However, a contending source can be located two hops away of the node that initially launches the signal. As in Fig. 2(b), they are two hops away from each other. In this specific case, the unaware competitor will access the channel as it does not receive the signal. To deal with this situation, if a node that has just finished transmitting (an ACK, for instance, in the case of Fig. 2(b)) receives an EIFS signal within about twice the maximum propagation delay (the time spent for a signal to reach the farthest node and to come back) from the transmission of the last bit, it is likely due to its last sending, then it rebroadcasts the signal in such a way that the competitor being two hops away is aware of the situation. In the case of Fig. 2(b)), the transmission of an ACK from node $D_{0}$ makes node $S_{1}$ to enter in EIFS deferment and to generate an EIFS signal. As node $D_{0}$ receives this signal and has just finished its transmission, it rebroadcasts it in such a way that node $S_{0}$ receives it and triggers an EIFS waiting period. EIFS delay synchronization is effective in despite of the fact that node $S_{0}$ is a two-hop away competitor (for $S_{1}$ ). The mechanism works when the EIFS signal is due to flow 1.

The results of the designed solution named FWM to the basic scenarios are represented in Table 4. Figures show that unfairness is no longer present for the two considered basic scenarios.

The integration of these new functionalities in legacy IEEE 802.11 concerns: (1) The introduction of a second signal of an impulse type. Like the first busy signal, the EIFS signal is only informative to state if either an EIFS waiting delay should be performed or not. (2) The triggering of an EIFS waiting period when an EIFS signal is received.

To sum up, a node requires two channels: a working channel running DCF and an out-of-band signaling channel. Minor modifications are performed in DCF: the consideration of the signaling channel for carrier sensing access method and the triggering of an EIFS waiting period if an EIFS signal is present.

\section{Evaluation of the proposed solution}

Jain's fairness index [6] is used to measure fairness. It is defined as follows:

$$
F=\frac{\left(\sum x_{i}\right)^{2}}{n\left(\sum x_{i}^{2}\right)}
$$

where $x_{i}$ is the share of flow $i$ and $n$ the number of flows. A totally fair allocation has a fairness index of 1 and totally 


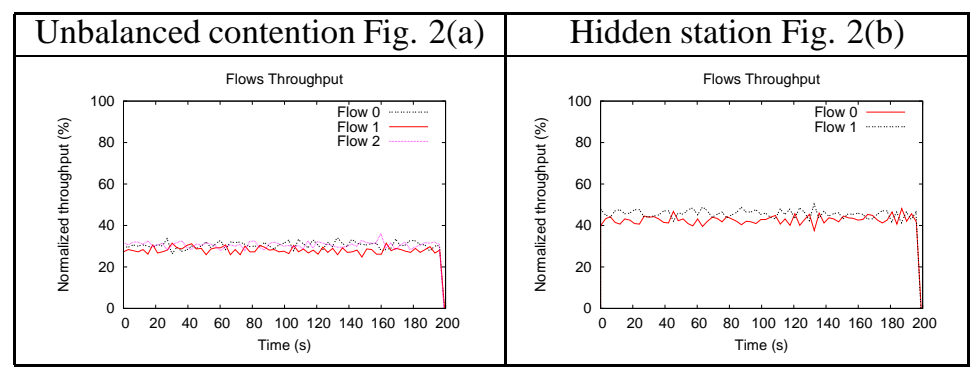

Table 4. Flows throughput with FWM

unfair allocation, a fairness index of $\frac{1}{n}$. The sum $\sum x_{i}$ represents the total throughput of the network and the standard variation $\sigma$, its variability. The evaluation results of the basic scenarios (Fig. 2) are presented in Table 5.

\begin{tabular}{|c|c|c|c|c|c|c|}
\hline \multirow{2}{*}{ Case } & \multicolumn{3}{|c|}{ Classical DCF } & \multicolumn{3}{|c|}{ FWM } \\
\cline { 2 - 7 } & $\sum x_{i}$ & $\sigma$ & $F$ & $\sum x_{i}$ & $\sigma$ & $F$ \\
\hline Fig. 2(a) & 176.80 & 0.92 & 0.68 & 89.00 & 0.66 & 0.99 \\
Fig. 2(b) & 88.66 & 0.15 & 0.5 & 88.14 & 0.15 & 0.99 \\
\hline
\end{tabular}

\section{Table 5. Performance comparison for basic unfair scenarios}

The results show that the proposed solution FWM is efficient to provide fairness in the studied cases while ensuring a stable throughput. The fairness index in the two scenarios is close to 1, which expresses a fair allocation. In scenario Fig. 2(b), the total bandwidth usage remains almost the same, but in scenario Fig. 2(a) it falls to about half of its value. Indeed, with DCF, the two side emitters can transmit simultaneously and the central pair is starved. It is like having two different links. The introduction of FWM can be viewed as constituting a single link. It is then obvious that bandwidth usage decreases by a factor of 2 . That constitutes the well-known trade-off between efficiency and fairness. A gain in fairness reduces the efficiency and inversely, a good efficiency leads to an unfair share of the resources. Now, let's evaluate this scheme in other scenarios (Fig. 3). The following scenarios are combinations of the basic cases. All conditions remain the same. The results for a classical network with only DCF and with the proposed solution FWM are shown in Table 6.

Fig. 3(a) represents a common illustration of the EIFS problem ([14], [5]). Node $S_{1}$ defers its transmission almost all the time due to that of $I_{10}$, because of its incapacity to decode it. Flow 1 is impacted. Indeed, node $D_{0}$ is comprised within the sensing range of node $S_{1}$ but is out of its transmission range. So, when node $D_{0}$ sends ACK in reply to node $I_{10}$ 's data packet, node $S_{1}$ receives the transmission

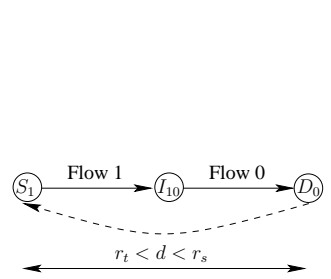

(a)

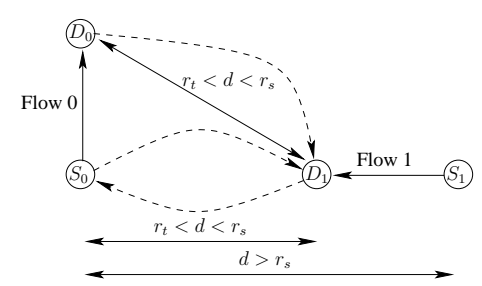

(b)

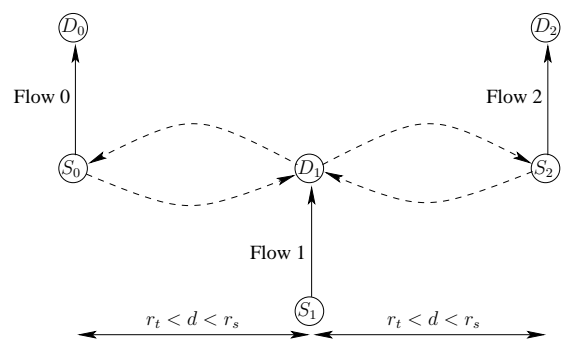

(c)

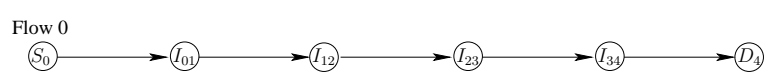

(d)

Figure 3. Other unfair scenarios

but cannot decode it. Then, it freezes for an EIFS (that is much larger than a DIFS) while node $I_{10}$ waits only a DIFS before accessing the channel. Then, node $I_{10}$ will access, more often than node $S_{1}$ as shown in Table 6 . The introduction of FWM solves efficiently this problem.

In Fig. 3(b), node $S_{1}$ is an hidden station to nodes $S_{0}$ and $D_{0}$, and when node $D_{1}$ receives successfully a packet from node $S_{1}$, it sends an ACK generating an EIFS at node $S_{0}$. In classical DCF, the hidden station effect is stronger than EIFS issue caused by ACK generation as $D_{1}$ experiences rarely a successful reception. Flow 1 is totally starved and 
cannot even get a portion of the bandwidth, corresponding to a fairness index equals to $\frac{1}{n}=0.5$. When FWM is applied, fairness is present while total bandwidth utilization remains the same.

In Fig. 3(c), node $S_{1}$ is a hidden station to both nodes $S_{0}$ and $S_{2}$. Thus, $D_{1}$ encounters interferences from nodes $S_{0}$ and $S_{2}$. When node $D_{1}$ receives a packet from node $S_{1}$, it makes nodes $S_{0}$ and $S_{2}$ wait for an EIFS. In a classical network, such interaction between flows cause the network to be unstable, but the fairness index equals 1 in average. With FWM applied, the network reaches fairness and stability but total bandwidth usage reduces from 118 to 89 . In addition to the fairness-efficiency trade-off, the stability-efficiency trade-off appears as for DCF, the standard variation $\sigma$ equals 37 with DCF and falls to less than 1 with FWM.

In Fig. 3(d), node $S_{0}$ sends traffic to node $D_{4}$. Intra-flow contention [17] exists between nodes, which are in interference range of each other, along the path. This results in an unstable behavior of the throughput for the classical case. When FWM is used, the instability disappears while the bandwidth usage remains the same. More coordination exists between nodes.

\begin{tabular}{|c|c|c|c|c|c|c|}
\hline \multirow{2}{*}{ Case } & \multicolumn{3}{|c|}{ Classical DCF } & \multicolumn{3}{c|}{ FWM } \\
\cline { 2 - 7 } & $\sum x_{i}$ & $\sigma$ & $F$ & $\sum x_{i}$ & $\sigma$ & $F$ \\
\hline Fig. 3(a) & 88.72 & 0.24 & 0.65 & 87.97 & 0.30 & 0.99 \\
Fig. 3(b) & 88.66 & 0.15 & 0.5 & 86.61 & 0.12 & 0.99 \\
Fig. 3(c) & 118.88 & 37.60 & 0.89 & 89.04 & 0.68 & 0.99 \\
Fig. 3(d) & 16.51 & 2.16 & - & 15.64 & 0.83 & - \\
\hline
\end{tabular}

\section{Table 6. Performance comparison for other unfair scenarios}

These results show all fairness and stability when the FWM scheme is applied. As expected, simulations with various packet sizes result in the same conclusion [8] .

\section{Existing solutions}

Some recent papers address the fairness problem in IEEE 802.11-based networks. In [3], modifications to backoff mechanisms are proposed for Wireless Local Area Networks (WLANs). Stations rate is ruled by an AIMD (Additive Increase Multiplicative Decrease) policy. A successful transmission leads to an increase of the rate while a failure makes the rate decrease. Some heuristics are given to choose suitable values for AIMD parameters. This estimation supposes the knowledge of the expected goodput or the number of stations, for example. Heusse et al [10] remove the backoff part of IEEE 802.11 and tackle with congestion avoidance by tuning the contention window. This tuning is based on idle slots observed by stations. While this mechanism exhibits better performance compared to DCF, it has not been tested for ad hoc configurations and does not take specific ad hoc issues into consideration. A probabilistic NAV (Network Allocation Vector) is added to IEEE 802.11 in [4] if the station is monopolizing the network. An opportunity is given to unfavoured stations to transmit. Still, the mechanism relies on parameters values whose tuning is not presented in the paper. Bruno et al [2] propose to insert an additional transmission control to backoff mechanism. When the backoff timer reaches 0 , a probability of transmission is computed by the sending station. This value is determined by the slot utilization [1] and by station usage of the bandwidth. While these schemes show improvements in fairness and channel utilization, the entire information asymmetry is not directly addressed. The computation of the slot utilization supposes that the packet length distribution is known. Recently, a deterministic method was proposed in [15] to provide fairness in 802.11 ad hoc networks. It inserts a waiting time based on the sharing (or not) of the channel. For some configurations the protocol performs well, but for some others as the one presented in in Fig. 2c, the unfairness problem still persists. Besides, the choice of parameters values depending on considered topology makes its implementation more difficult. EHATDMA [9] addresses fairness issues by using an hybrid asynchronous time division multiple access, and considering their main causes separately. Even if performance results are very promising, its complexity and the use of TDMA make its implementation in 802.11 technology hard. Emerging standards for Wireless Mesh technology are presented in [12]. Task group IEEE 802.11s studies enhancements for this type of networks to facilitate multi-channel operations for single radio devices [13]. Nodes utilize a common channel for the selection of a free channel for data communication. That reduces the contention among nodes and improves performance. However, this approach is sensitive to network density. Indeed, with a large number of nodes in contention, the number of channels is not enough, fairness issues can still appear within data channels.

To sum up, all the previous solutions require either the tuning of different parameters or a knowledge of the underlying network or the traffic pattern. Besides, the implementation of most of them in real world scenario introduces too many modifications in original DCF access.

\section{Conclusion}

This paper presents a simple Fair Wireless MAC (FWM) for IEEE 802.11-based ad hoc networks. It aims at providing fairness in wireless ad hoc networks independently on the location of nodes within the network.

Three main causes of unfairness are identified and clas- 
sified into two categories: the lack of synchronization and EIFS issue. The solution consisting in the propagation of neighboring information and a balanced waiting time is proposed. An additional dedicated channel is required by FWM. When a node is in a receiving state, it continuously transmits on the signaling channel. Carrier sensing takes this signal into consideration for the determination of the medium state. However, this mechanism is not sufficient to solve the EIFS issue caused by differences in sensing and transmission ranges. The solution is to trigger synchronously an EIFS wait period. A node entering an EIFS deferment generates a short signal (an impulse). Each node receiving the impulse will also start an EIFS wait period. The last transmitter occupying the channel rebroadcasts the signal if that is due to its last transmission. FWM proposes to combine CSMA/CA with the busy tone scheme to provide fairness. Extensive simulations on NS-2 show the efficiency of the proposed protocol in various scenarios. From the implementation point of view, this solution is feasible because IEEE 802.11 allows three separate channels. The main advantages of FWM come from its simplicity and because it does not require additional computation capability. Also, it supposes no knowledge on the traffic or the topology and it does not rely on parameters others than that of IEEE 802.11, making it flexible for various configurations. The nature and operation of basic DCF are kept as only minor modifications are performed to which stability of the protocol is attributed. The main drawback of the proposed solution is the use of an additional signaling channel. However, the authors are convinced that nodes resynchronization cannot be efficiently performed by a temporal mechanism; a spatial mechanism, namely a dedicated channel and signal are necessary. Implementation constrains are minor compared to the advantages provided to the communications in ad hoc networks.

\section{References}

[1] L. Bononi, M. Conti, and E. Gregori. Runtime Optimization of IEEE 802.11 Wireless LANs Performance. IEEE Transactions on Parallel and Distributed Systems, 15(1):66-80, January 2004.

[2] R. Bruno, C. Chaudet, M. Conti, and E. Gregori. A Novel Fair Medium Access Control for 802.11-based Multi-Hop Ad hoc Networks. In Proceedings of the 14th IEEE Workshop on Local and Metropolitan Area Networks (LanMan) Chania, Crete, Greece, September 2005.

[3] S. Cai, Y. Liu, and W. Gong. Analysis of An AIMD Based Collision Avoidance Protocol in Wireless Data Networks. In Proceedings of IEEE Conference on Decision and Control (CDC), Hawaii, USA, December 2003.

[4] C. Chaudet, G. Chelius, H. Meunier, and D. Simplot-Ryl. Adaptive Probabilistic NAV to Increase Fairness in Ad Hoc 802.11 MAC Layer. In Proceedings of the Fourth annual
Mediterranean workshop on Ad Hoc Networks (Med-HocNet 2005) - Island of Porquerolles, France, June 2005.

[5] C. Chaudet and D. D. I. G. Lassous. Performance Issues with IEEE 802.11 in Ad Hoc Networking. IEEE Communications Magazine, 43(7), July 2005.

[6] D.-M. Chiu and R. Jain. Analysis of the Increase and Decrease Algorithm for Congestion Avoidance in Computer Networks. Computer Networks and ISDN Systems, 17:1-14, 1989.

[7] I. Guerin-Lassous. Quelles performances pour les rseaux WiFi ? Techniques de l?Ingnieur, TE 7381, May 2005.

[8] F. Harivelo and P. Anelli. A Simple Fair Wireless Medium Access for 802.11-based Ad Hoc Networks. Technical report, Technical Report 0601-1, http://www.univreunion.fr/ harivelo, IREMIA, Université de La Réunion, France, April, 102006.

[9] J. He and H. K. Pung. Fairness of medium access control protocols for multi-hop ad hoc wireless networks. Computer Networks, 48(6):867-890, August 2005.

[10] M. Heusse, F. Rousseau, R. Guillier, and A. Duda. Idle Sense: An Optimal Access Method for High Throughput and Fairness in Rate Diverse Wireless LANs. In ACM SIGCOMM, 2005.

[11] IEEE 802.11 WG. Wireless LAN medium access control (MAC) and physical layer (PHY) specifications. In IEEE standard 802.11, 1999 Edition, 1999.

[12] M. J. Lee, J. Zheng, Y.-B. Ko, and D. M. Shrestha. Emerging Standards for Wireless Mesh Technology. IEEE Wireless Communications, 13(2):56-63, April 2006.

[13] S.-W. Lee, J. Lee, C. Zhu, and R. Taori. 802.11 TGs MAC Enhancement Proposal. IEEE 802 11-05/0589r0, June 2005.

[14] Z. Li, S. Nandi, and A. K. Gupta. ECS: an Enhanced Carrier Sensing Mechanism for Wireless Ad-hoc Networks. Computer Communication, 28(17):1970-1984, October 2005.

[15] T. Razafindralambo and I. Guerin-Lassous. Increasing Fairness and Efficiency using the MadMac Protocol in Ad Hoc Networks. In Networking 2006, Coimbra, Portugal, 2006.

[16] F. A. Tobagi and L. Kleinrock. Packet Switching in Radio Channels: Part II-The Hidden Terminal Problem in Carrier Sense Multiple-Access and the Busy-Tone Solution. IEEE Transactions on Communication, 23(12):1417-1433, 1975.

[17] H. Zhai, J. Wang, and Y. Fang. Distributed Packet Scheduling for Multihop Flows in Ad Hoc Networks. IEEE Wireless Communications and Networking Conference (WCNC'04), Atlanta, Georgia, March 2004. 